\title{
An Adaptive Sliding Mode Control Scheme for Grid Integration of a PV System
}

\author{
Aurobinda Bag, Bidyadhar Subudhi, and Pravat Kumar Ray
}

\begin{abstract}
An Adaptive Sliding Mode Control (ASMC) algorithm is developed in this paper for grid synchronization of a photovoltaic (PV) system. This ASMC algorithm minimizes the difference between the reference inverter current and the actual inverter current of the grid connected PV system (GCPVS) during unbalanced loading, grid voltage distortion and variation in solar irradiance. The maximum output power from the PV panel is obtained using modified incremental conductance (IC) MPPT algorithm. Instantaneous power theory (IPT) is employed to transfer active power and reactive power between the PV system, grid and the non-linear load. The ASMC-IC-IPT control scheme is applied to a grid connected PV system firstly using simulation and then on a prototype experimental setup. The performance of ASMC-IC-IPT scheme is compared with that of sliding mode control (SMC)-IC-IPT scheme. From the obtained results, it is observed that ASMC-IC-IPT control scheme outperforms the SMC-IC-IPT control scheme during transient, steady state and dynamic loading conditions.
\end{abstract}

Index Terms-Adaptive sliding mode control, grid synchronization, Lyapunov function, total harmonic distortion.

\section{INTRODUCTION}

$\mathrm{I}_{\mathrm{n}}^{\mathrm{N}}$ $\mathrm{N}$ view of the load disturbance and uncertainties in parameters e.g. voltage, current, resistance, inductance of a grid connected PV system, designing an appropriate control algorithm is important. Also a number of non-linear control algorithms have been proposed for a PV system such as Lyapunov based controller [1], two-loop controller, SMC controller [2]. Among the aforesaid non-linear control algorithms, SMC algorithm is popular due to its robustness in face of parametric uncertainties and load variations. However, the SMC algorithm yields chattering in the control signal resulting reduction in control accuracy and high heating losses in the power circuit [3]. Sliding mode observer based adaptive slip ratio control algorithm is proposed in [4] for a hybrid electric vehicle. In [5], the adaptive global fast terminal SMC using fuzzy neural network approaches are proposed for an H-bridge inverter. An adaptive fuzzy SMC [6] is proposed for a two stage single-phase grid connected PV system. An adaptive SMC is also developed for a single-phase grid connected PV system in [7].

The contribution of this paper lies in designing an ASMCIC-IPT scheme for a three-phase grid connected PV system. The robustness of the ASMC-IC-IPT scheme is investigated

\footnotetext{
Manuscript received June 7, 2018.

Authors are with the Department of Electrical Engineering, National Institute of Technology, Rourkela 769008, India (e-mail: bidyadhar@nitrkl.ac.in; aurobinda.bag80@gmail.com; rayp@nitrkl.ac.in).

Digital Object Identifier 10.24295/CPSSTPEA.2018.00035
}

over SMC-IC-IPT scheme. The performance of the ASMC-ICIPT is evaluated in both simulation and an experimental setup with grid voltage distortion, load variation and change in solar irradiance.

The rest of the paper is organised as follows. The modified incremental conductance MPPT algorithm is presented in Section II. In Section III, the proposed adaptive SMC scheme is presented that considers uncertainties due to parameter variation and measurements. Section IV describes the instantaneous power theory to estimate the reference inverter current. In Section V, simulation results and discussion are presented. Section VI presents the experimental results with discussion. Section VII provides the conclusion of the paper.

\section{Adaptive Sliding Mode Control Algorithm}

The schematic diagram of a GCPVS is shown in Fig. 1. The nonlinear loads I and II are used to verify the performance of ASMC-IC-IPT scheme, as shown in Fig. 1. A modified IC MPPT algorithm is used to extract maximum power from PV panel [8].

\section{A. Modeling of Grid Connected PV System}

The dynamic model of the GCPVS can be derived using Fig. 1.

$$
\begin{gathered}
\frac{d}{d t} I_{c d}=\frac{1}{L_{c}} D_{d} V_{p v}-\frac{R_{c}}{L_{c}} I_{c d}+w I_{c q}-\frac{1}{L_{c}} V_{g d} \\
\frac{d}{d t} I_{c q}=\frac{1}{L_{c}} D_{q} V_{p v}-\frac{R_{c}}{L_{c}} I_{c q}-w I_{c d}-\frac{1}{L_{c}} V_{g q} \\
\frac{d}{d t} V_{p v}=\frac{1}{C_{d c}} I_{p v}-\frac{1}{C_{d c}}\left(D_{d} I_{c d}+D_{q} I_{c q}\right)
\end{gathered}
$$

where, $I_{c d}, I_{c q}$ are the d-q axis VSI currents, $V_{g d}, V_{g q}$ are $d-q$ axis grid voltages, $D_{d}, D_{q}$, are $d$ - $q$ axes duty cycle and $w$, represents the frequency of the grid voltage.

\section{B. Assumptions}

In the GCPVS, there may be some unpredictable electromagnetic interference during the operation which causes noise while measuring the parameters such as voltage, current through sensors. Also the parameters such as resistance, inductance of inverter interface may vary due to variation in ambient temperature and skin effect. To represent the actual value of these parameters e.g. voltage, current, resistance and inductance which are used by the controller, the following assumptions are taken. 


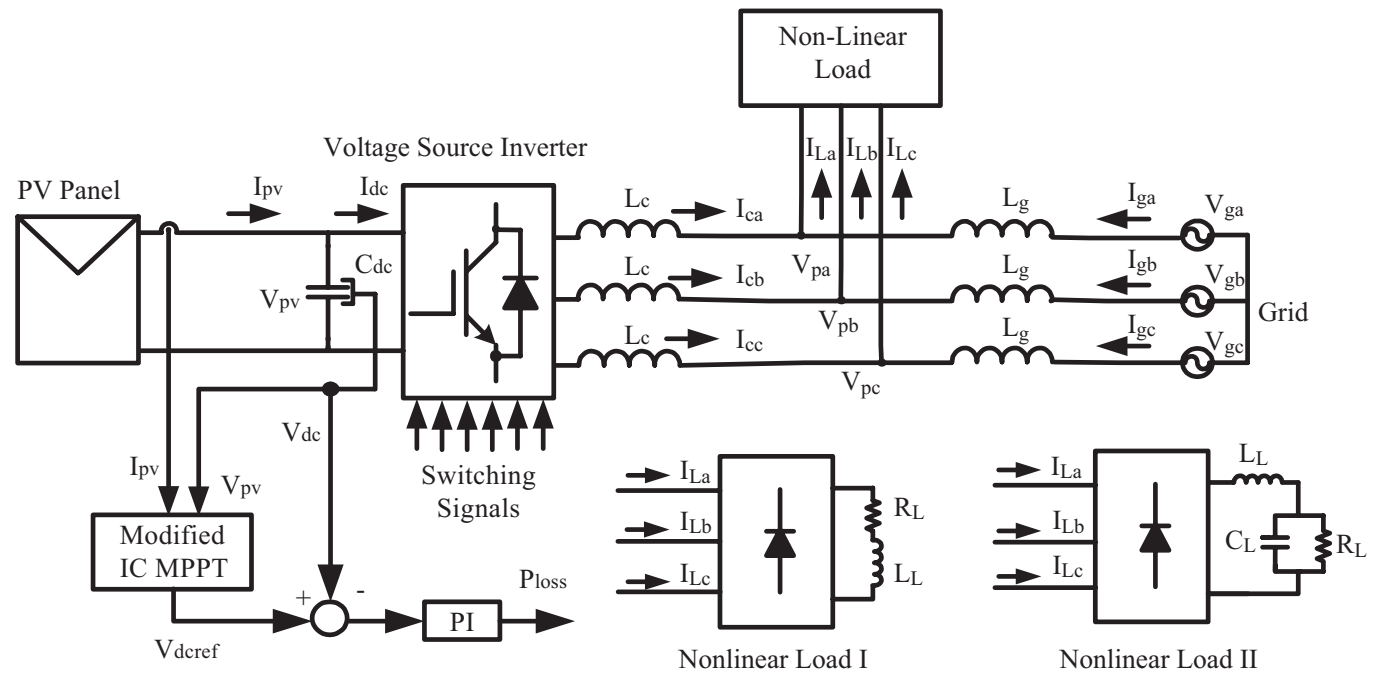

Fig. 1. Schematic Diagram for grid connected PV System.

$$
\begin{gathered}
\left|I_{c d}-I_{c d, \text { meas }}\right| \leqslant \Delta I_{c d}, I_{c d} \leqslant I_{c d, \text { meas }}+\Delta I_{c d} \\
\left|I_{c q}-I_{c q, \text { meas }}\right| \leqslant \Delta I_{c q}, I_{c q} \leqslant I_{c q, \text { meas }}+\Delta I_{c q} \\
\left|\frac{V_{g d}}{L_{c}}-\frac{V_{g d, \text { meas }}}{L_{c, \text { meas }}}\right| \leqslant \Delta \frac{V_{g d}}{L_{c}} \\
\left|\frac{V_{g q}}{L_{c}}-\frac{V_{g q, \text { meas }}}{L_{c, \text { meas }}}\right| \leqslant \Delta \frac{V_{g q}}{L_{c}} \\
\mid K_{d} e_{d}-K_{d} e_{d, e s t} \leqslant K_{d} \Delta e_{d} \\
\mid K_{q} e_{q}-K_{q} e_{q, \text { est }} \leqslant K_{q} \Delta e_{q} \\
\left|K_{1} I_{c d}-K_{1, \text { est }} I_{c d \text { meas }}\right| \leqslant \Delta K_{1} I_{c d} \\
\left|K_{2} I_{c q}-K_{2, \text { est }} I_{c q, \text { meas }}\right| \leqslant \Delta K_{2} I_{c q}
\end{gathered}
$$

where $R_{c, \text { meas }}, L_{c, \text { meas }}, I_{c d, \text { meas }}, I_{c q, \text { meas }}, V_{\text {gd, meas }}, V_{\text {gq, meas }}$ represent the measured values, $K_{1, e s t}, \mathrm{~K}_{2, \text { est }}, e_{d, \text { est }}, e_{q, \text { est }}$ represent the estimated values and $\Delta I_{c d}, \Delta I_{c q}, \Delta \frac{V_{g d}}{L_{c}}, \Delta \frac{V_{g q}}{L_{c}}, \Delta e_{d}, \Delta e_{q}, \Delta K_{1} I_{c d}$, $\Delta K_{2} I_{c q}$ are the uncertainties due to parametric variations and measurements.

\section{Design of Adaptive Sliding Mode Control Scheme}

It is intended to design an adaptive SMC algorithm such that tracking of the reference inverter current is achieved. It is necessary that the magnitude, phase and frequency of the actual inverter current should be equal to the reference inverter current. We choose sliding surfaces as follows

$$
S=\left[\begin{array}{c}
S_{d} \\
S_{q}
\end{array}\right]
$$

where $S_{d}, S_{q}$ denote sliding surfaces for $d$ and $q$ axes. In order to maintain the enhanced transient response and to reduce the steady state error, we introduce the integral forms as follows

$$
\left[\begin{array}{l}
S_{d} \\
S_{q}
\end{array}\right]=\left[\begin{array}{c}
e_{d}+K_{d} \int_{-\infty}^{t} e_{d}(\tau) d \tau+e_{d 0} \\
e_{q}+K_{q} \int_{-\infty}^{t} e_{q}(\tau) d \tau+e_{q 0}
\end{array}\right]
$$

where $K_{d}, K_{q}$ are positive constants, $e_{d 0}, e_{q 0}$ are initial errors between the reference and the actual $d-q$ components of inverter current. $e_{d}$ and $e_{q}$ are defined as follows

$$
e_{d}=I_{c d}^{*}-I_{c d}, e_{q}=I_{c q}^{*}-I_{c q}
$$

So $S_{d}=0$ and $S_{q}=0$ represent the precise tracking of $d-q$ components of inverter current of GCPVS.

When the system state reaches the sliding manifolds, the structure of the feedback loop is adaptively altered to slide the system state along the sliding surface. The time derivative of (13) yields

$$
\begin{aligned}
\dot{S}_{d}=\frac{d}{d t} S_{d} & =\frac{d}{d t}\left(I_{c d}^{*}-I_{c d}\right)+K_{d}\left(I_{c d}^{*}-I_{c d}\right) \\
& =-K_{d 1} \operatorname{sign}\left(S_{d}\right) \\
\dot{S}_{q}=\frac{d}{d t} S_{q} & =\frac{d}{d t}\left(I_{c q}^{*}-I_{c q}\right)+K_{q}\left(I_{c q}^{*}-I_{c q}\right) \\
& =-K_{q 1} \operatorname{sign}\left(S_{q}\right) .
\end{aligned}
$$

Substituting (1) and (2) into (14), yields

$$
\begin{gathered}
\frac{d}{d t} S_{d}=\frac{d}{d t} I_{c d}^{*}-\frac{1}{L_{c}} D_{d} V_{p v}+\frac{R_{c}}{L_{c}} I_{c d}-w I_{c q} \\
+\frac{1}{L_{c}} V_{g d}+K_{d} e_{d} \\
\frac{d}{d t} S_{q}=\frac{d}{d t} I_{c q}^{*}-\frac{1}{L_{c}} D_{q} V_{p v}-\frac{R_{c}}{L_{c}} I_{c q}+w I_{c d} \\
+\frac{1}{L_{c}} V_{g q}+K_{q} e_{q}
\end{gathered}
$$


(15) can be rewritten as

$$
\frac{d}{d t} S=F+D U_{d q}
$$

where

$$
\begin{gathered}
F=\left[\begin{array}{c}
F_{d} \\
F_{q}
\end{array}\right]=\frac{d}{d t}\left[\begin{array}{c}
I_{c d}^{*} \\
I_{c q}^{*}
\end{array}\right]+\left[\begin{array}{cc}
\frac{R_{c}}{L_{c}} & -w \\
w-\frac{R_{c}}{L_{c}}
\end{array}\right]\left[\begin{array}{c}
I_{c d} \\
I_{c q}
\end{array}\right] \\
+\left[\begin{array}{cc}
\frac{1}{L_{c}} & 0 \\
0 & \frac{1}{L_{c}}
\end{array}\right]\left[\begin{array}{c}
V_{g d} \\
V_{g q}
\end{array}\right]+\left[\begin{array}{cc}
K_{d} & 0 \\
0 & K_{q}
\end{array}\right]\left[\begin{array}{c}
e_{d} \\
e_{q}
\end{array}\right] \\
D=\left[\begin{array}{cc}
-\frac{V_{p v}}{L_{c}} & 0 \\
0 & -\frac{V_{p v}}{L_{c}}
\end{array}\right] \text { and } \mathrm{U}_{d q}=\left[\begin{array}{c}
D_{d} \\
D_{q}
\end{array}\right] .
\end{gathered}
$$

The control law can be obtained as follows

$$
U_{d q}=-D^{-1}\left\{\left[\begin{array}{c}
F_{d,} \\
F_{q,}
\end{array}\right]+\left[\begin{array}{cc}
K_{d 1} & 0 \\
0 & K_{q 1}
\end{array}\right]\left[\begin{array}{l}
\operatorname{sign}\left(S_{d}\right) \\
\operatorname{sign}\left(S_{q}\right)
\end{array}\right]\right\}
$$

The switching action in (17) may yield chattering owing to presence of sign functions. This can be minimized by introducing a boundary layer. This can be obtained by replacing the sign function by a saturation function $\operatorname{sat}(S)$ with narrow region of the sliding surface through which the discontinuities in the switching control action can be avoided.

$$
\operatorname{sat}(S)=\frac{S}{|S|+\lambda}
$$

where $\lambda<<|S|$ is a small positive gain i.e. the width of the boundary layer.

In order to make the SMC algorithm adaptive considering the uncertainties due to parametric variations and measurements as described in Section III-A, the control law can be derived as follows

$$
U_{d q}=-D^{-1}\left\{\left[\begin{array}{l}
F_{d, e s t} \\
F_{q, e s t}
\end{array}\right]+\left[\begin{array}{cc}
K_{d 1} & 0 \\
0 & K_{q 1}
\end{array}\right]\left[\begin{array}{l}
\operatorname{sat}\left(S_{d}\right) \\
\operatorname{sat}\left(S_{q}\right)
\end{array}\right]\right\}
$$

where

$$
\begin{gathered}
F_{e s t}=\left[\begin{array}{c}
F_{d, e s t} \\
F_{q, e s t}
\end{array}\right]=\frac{d}{d t}\left[\begin{array}{c}
I_{c d}^{*} \\
I_{c q}^{*}
\end{array}\right] \\
+\left[\begin{array}{cc}
K_{1, e s t} & -w \\
w & K_{2, e s t}
\end{array}\right]\left[\begin{array}{c}
I_{c d} \\
I_{c q}
\end{array}\right]+\left[\begin{array}{cc}
\frac{1}{L_{c}} & 0 \\
0 & \frac{1}{L_{c}}
\end{array}\right]\left[\begin{array}{c}
V_{g d} \\
V_{g q}
\end{array}\right] \\
+\left[\begin{array}{cc}
K_{d} & 0 \\
0 & K_{q}
\end{array}\right]\left[\begin{array}{c}
e_{d} \\
e_{q}
\end{array}\right],
\end{gathered}
$$

$$
D=\left[\begin{array}{cc}
-\frac{V_{p v}}{L_{c}} & 0 \\
0 & -\frac{V_{p v}}{L_{c}}
\end{array}\right] \text { and } \mathrm{U}_{d q}=\left[\begin{array}{c}
D_{d} \\
D_{q}
\end{array}\right]
$$

Accordingly the tunning law for $d$-axis can be derived as follows

$$
\dot{K}_{1, \text { est }}=\frac{I_{c d, \text { meas }} S}{\alpha}
$$

and for $q$-axis (see the Appendix) is as follows

$$
\dot{K}_{2, \text { est }}=\frac{I_{c q, \text { meas }} S}{\alpha}
$$

where $K_{1}=\frac{R_{c}}{L_{c}}$. The sensitivity of the controlled system to variation of uncertainties and external disturbances still exists in the reaching phase, before sliding mode occurs, which means that the loss of robustness in the transient state. To guarantee the sliding mode at the initial time instant, the initial conditions of the integrators should be chosen as

$$
I_{d 0}=\frac{-e_{d 0}}{K_{d}} \text { and } I_{q 0}=\frac{-e_{q 0}}{K_{q}}
$$

where $I_{d 0}$ and $I_{q 0}$ are the initial conditions of the integrator defined as

$$
I_{d 0}=\int_{-\infty}^{0} e_{d}(\tau) d \tau, I_{q 0}=\int_{-\infty}^{0} e_{q}(\tau) d \tau
$$

Hence at $t=0$

$$
\begin{gathered}
S_{d 0}=e_{d 0}+K_{d} I_{d 0}=0 \\
S_{q 0}=e_{q 0}+K_{q} I_{q 0}=0
\end{gathered}
$$

(23) and (24) imply that the system states are on the sliding surfaces at the initial time instant without the reaching phase, and the complete robustness can be obtained during the entire response. Fig. 2 shows the structure of the proposed ASMC employed with the instantaneous power theory for the GCPVS. The reference active power and reactive power for VSI are calculated using the instantaneous power theory. Then the reference inverter current is calculated using (32) and (33). After implementation of ASMC, the required gate signal is generated using (18), which is applied to the VSI.

\section{Reference CuRRENT GenERATION FOR VSI}

We employed IPT and power balance to generate the reference current for VSI. The $\alpha, \beta$ components of grid voltage, grid current and load current can be calculated using Clarke transformation as follows 


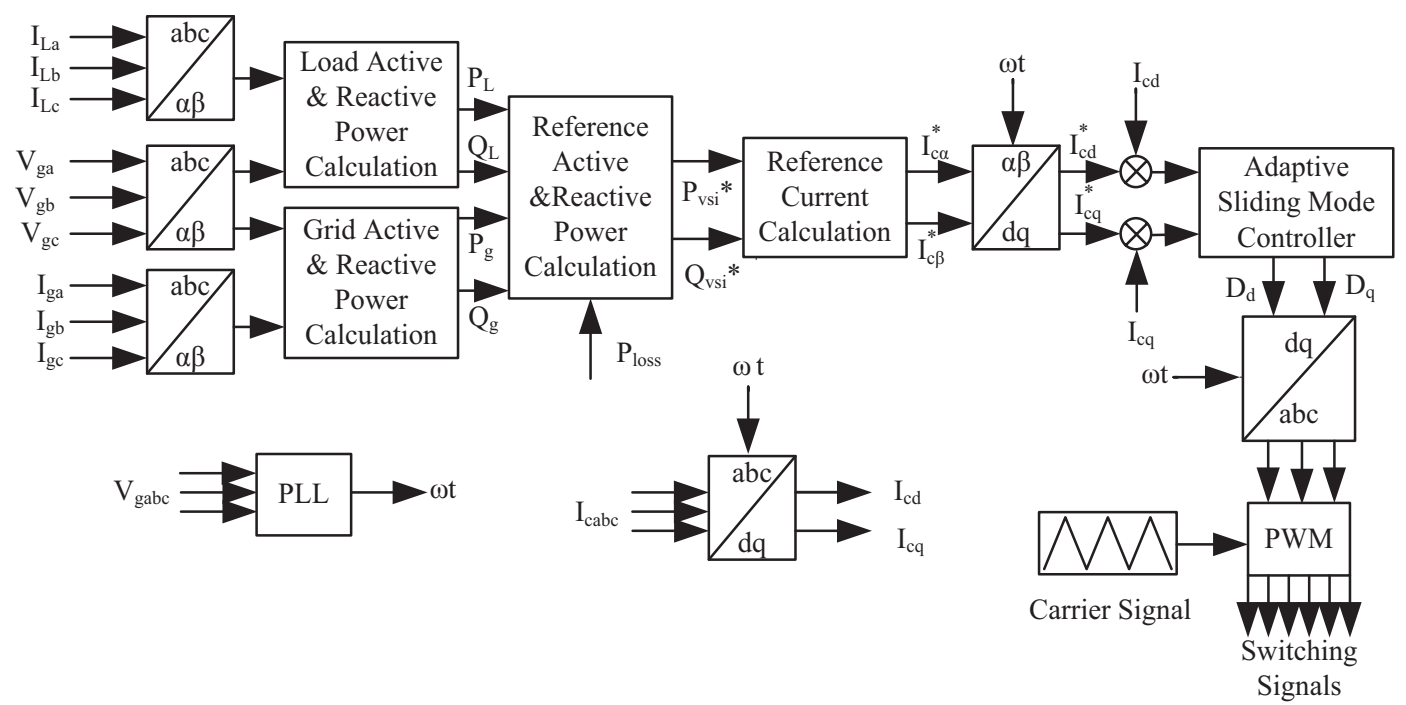

Fig. 2. Structure of adaptive SMC algorithm for a grid connected PV System.

$$
\begin{aligned}
& {\left[\begin{array}{c}
V_{g \alpha} \\
V_{g \beta}
\end{array}\right]=M\left[\begin{array}{l}
V_{g a} \\
V_{g b} \\
V_{g c}
\end{array}\right]} \\
& {\left[\begin{array}{c}
I_{L \alpha} \\
I_{L \beta}
\end{array}\right]=M\left[\begin{array}{c}
I_{L a} \\
I_{L b} \\
I_{L c}
\end{array}\right]} \\
& {\left[\begin{array}{c}
I_{g \alpha} \\
I_{g \beta}
\end{array}\right]=M\left[\begin{array}{c}
I_{g a} \\
I_{g b} \\
I_{g c}
\end{array}\right]}
\end{aligned}
$$

where, $M=\sqrt{\frac{2}{3}}\left[\begin{array}{c}1-\frac{1}{2}-\frac{1}{2} \\ 0 \frac{\sqrt{3}}{2}-\frac{\sqrt{3}}{2}\end{array}\right]$

TABLE I

PARAMETERS FOR ASMC SCHEME

\begin{tabular}{cccc}
\hline \hline Parameter & Value & Parameter & Value \\
\hline$K_{d}$ & 100 & $K_{q}$ & 100 \\
$K_{d 1}$ & 1000 & $K_{q 1}$ & 1000 \\
\hline \hline
\end{tabular}

dc link capacitor voltage at a fixed value. The utility active power can be calculated as follows.

$$
P_{g}=V_{g \alpha} I_{g \alpha}+V_{g \beta} I_{g \beta} \text {. }
$$

If the $\left(P_{\text {loss }}\right)$ is supplied by the PV panel, then the reference real and reactive power for inverter is calculated as follows

$$
\begin{gathered}
P_{v s i}^{*}=P_{L}-\bar{P}_{g}+\bar{P}_{l o s s} \\
Q_{v s i}^{*}=Q_{l} .
\end{gathered}
$$

as follows

$$
\left[\begin{array}{c}
P_{L} \\
Q_{L}
\end{array}\right]=\left[\begin{array}{cc}
V_{\alpha} & V_{\beta} \\
-V_{\beta} & V_{\alpha}
\end{array}\right]\left[\begin{array}{c}
I_{\alpha} \\
I_{\beta}
\end{array}\right] .
$$

The real power and reactive power of the load comprises of an average component (superscript ${ }^{-}$) and an oscillating component (superscript $\sim$ ). The oscillating component of power can be obtained using a low pass filter (LPF) [9].

$$
\begin{gathered}
P_{L}=\bar{P}_{L}+\widetilde{P}_{L} \\
Q_{L}=\bar{Q}_{L}+\tilde{Q}_{L} .
\end{gathered}
$$

The dc link capacitor voltage of VSI is measured at the time of operation of VSI. A PI-controller is employed to regulate the dc link voltage. The reference dc link voltage is obtained as the output of the modified IC MPPT algorithm. The output from the PI-controller represents as power loss $\left(P_{\text {loss }}\right)$ which flows to (from) the capacitor $C_{d c}$ to keep the

Accordingly the reference d-q component of inverter current can be estimated by following equations.

$$
\begin{gathered}
{\left[\begin{array}{c}
I_{c \alpha}^{*} \\
* \\
I_{c \beta}^{*}
\end{array}\right]=\left[\begin{array}{cc}
V_{\alpha} & V_{\beta} \\
-V_{\beta} & V_{\alpha}
\end{array}\right]^{-1}\left[\begin{array}{c}
P_{v s i}^{*} \\
Q_{v s i}^{*}
\end{array}\right]} \\
{\left[\begin{array}{c}
I_{c d}^{*} \\
I_{c q}^{*}
\end{array}\right]=\left[\begin{array}{r}
\cos \varphi \sin \varphi \\
-\sin \varphi \cos \varphi
\end{array}\right]\left[\begin{array}{c}
I_{c \alpha}^{*} \\
I_{c \beta}^{*}
\end{array}\right] .}
\end{gathered}
$$

\section{Simulation Results}

The simulation is performed for GCPVS using both nonlinear load I and II with ASMC-IC-IPT scheme shown in Fig. 1 in MATLAB/SIMULINK. The performance of ASMC-IC-IPT scheme is compared with that of the SMCIC-IPT scheme [8] and summarized in Section V-C. The parameters used for the PV system are given in [8]. The control parameters are given in TABLE I. 


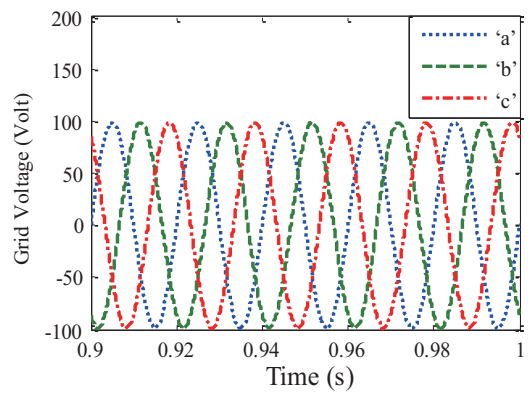

(a)

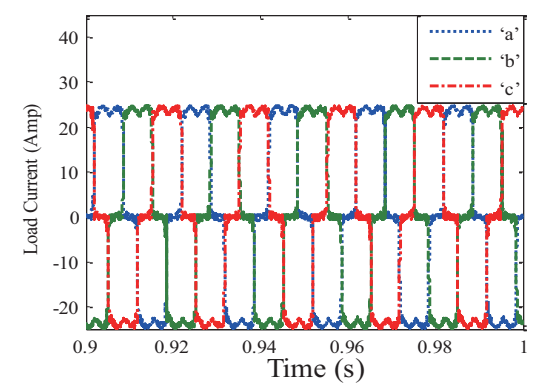

(d)

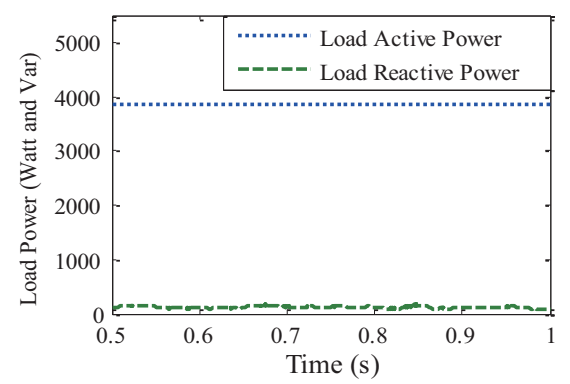

(g)

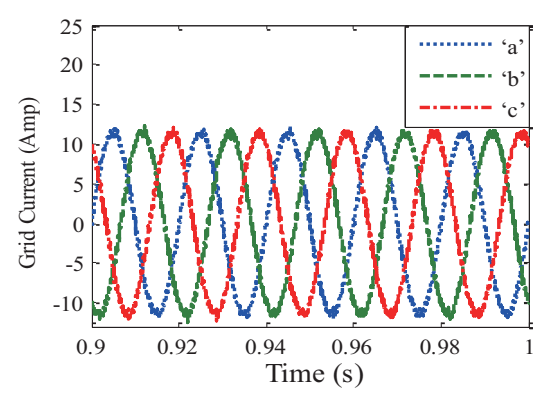

(b)

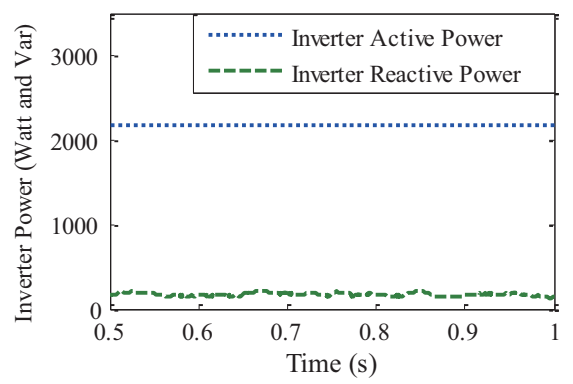

(e)

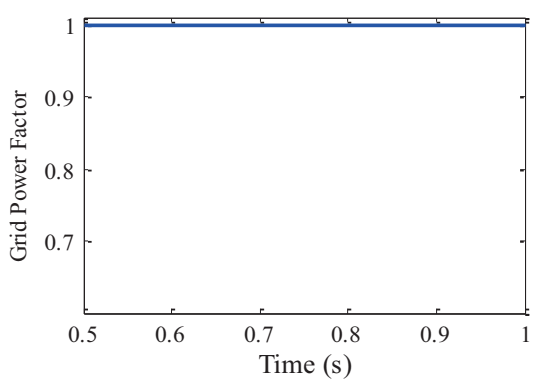

(h)

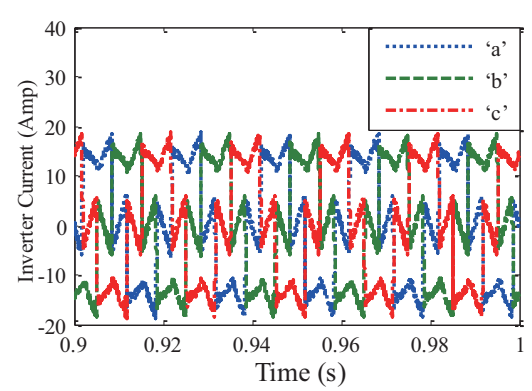

(c)

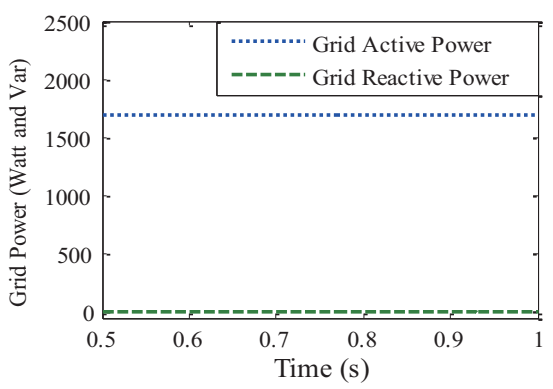

(f)

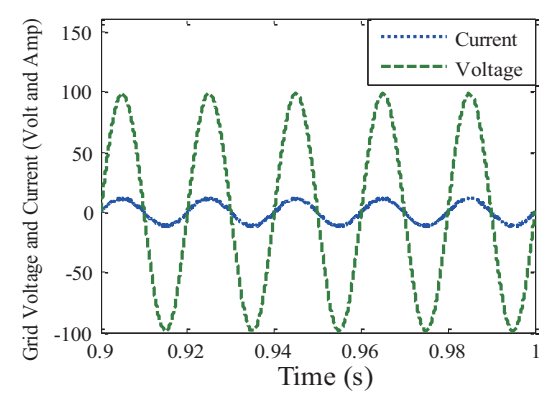

(i)

Fig. 3. Steady state performance of ASMC-IC-IPT using non-linear load I and ideal grid (with out grid voltage distortion) condition: (a) Grid voltage. (b) Grid current. (c) Inverter current. (d) Load current. (e) Inverter power. (f) Grid power. (g) Load power. (h) Grid power factor. (i) Grid voltage and current for phase 'a'.

\section{A. Performance of the Proposed ASMC-IC-IPT Using Non- Linear Load I}

The simulated performance of the proposed ASMCIC-IPT scheme using non-linear load I (Fig. 1) is shown in Fig. 3. The three-phase grid voltage $V_{g a}$, grid current $I_{g a}$, inverter current $I_{c a}$ and load current $I_{L a}$ for ASMC-ICIPT scheme are shown in Fig. 3(a), (b), (c) and (d). From Fig. 3(b), (c) and (d), it can be observed that, the total load current, 26.6 A is the summation of PV-VSI current, 15.1 A and the grid current, 11.5 A. The total load active power, $3.9 \mathrm{~kW}$, is the addition of PV-VSI output power, $2.2 \mathrm{~kW}$ and the grid power, $1.7 \mathrm{~kW}$, which is seen from Fig. 3(e), (f), and (g). The load reactive power, 200 VARs is supplied by PV-VSI system. Therefore, the grid power factor as shown in Fig. 3(h) is unity. The grid voltage and current for phase ' $a$ ' are in the same phase, which is seen from Fig. 3(i).

\section{B. Performance of the proposed ASMC-IC-IPT Using Non- Linear Load II}

The performance for ASMC-IC-IPT scheme using nonlinear load II (Fig. 1) is shown in Fig. 4. The three-phase grid current, grid voltage and current for phase 'a' and grid current THD are shown in Fig. 4(a), (b) and (c) respectively. It is seen from Fig. 4(c) that, the THD of grid current obtained in ASMC-IC-IPT scheme using non-linear load II is $2.82 \%$, which is slightly more than that obtained from Fig. 6(b) using non-linear load I. This may be due to inclusion of capacitor in the nonlinear load II.

\section{Performance of the ASMC-IC-IPT Under Grid Distortion}

The simulation results for ASMC-IC-IPT scheme under grid voltage distortion are shown in Fig. 5. In Fig. 5(a), (b) 


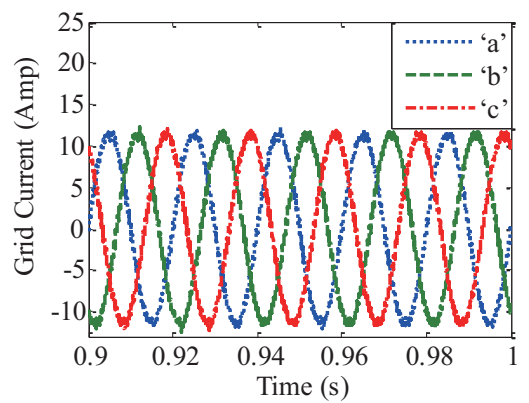

(a)

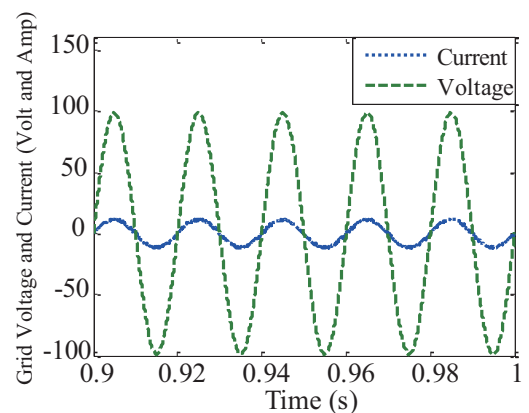

(b)

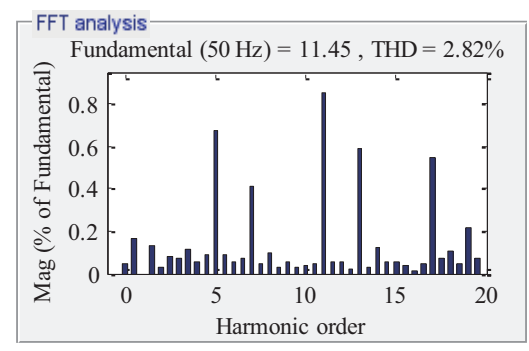

(c)

Fig. 4. Steady state performance of ASMC-IC-IPT using non-linear load II and ideal grid (with out grid voltage distortion) condition: (a) Grid current. (b) Grid voltage and current for phase 'a'. (c) Grid current THD.

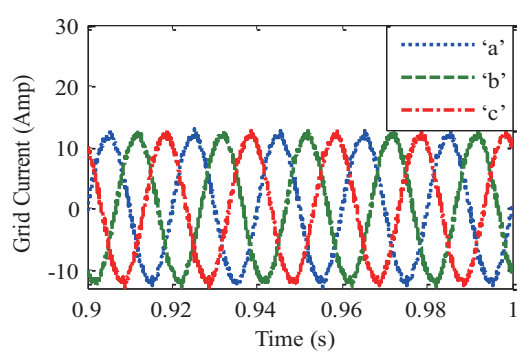

(a)

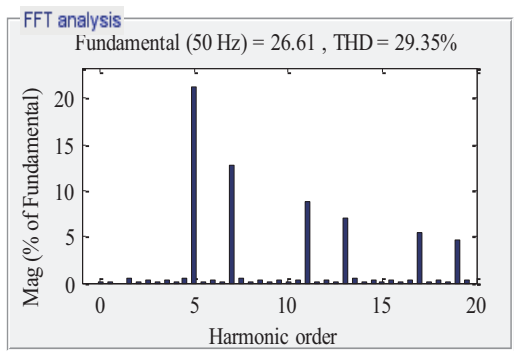

(d)

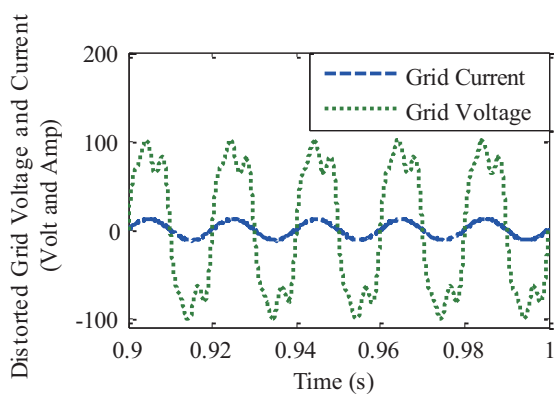

(b)

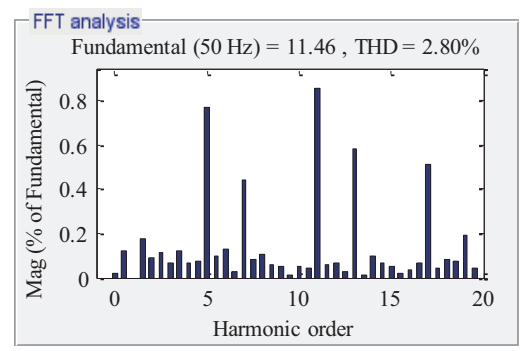

(e)

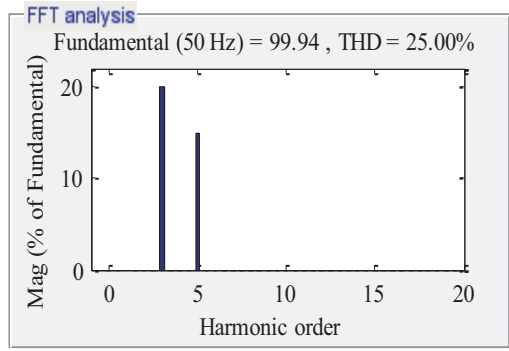

(c)

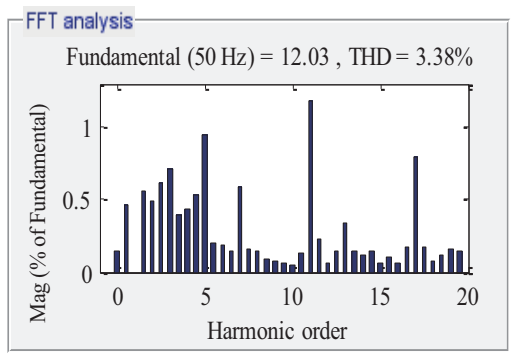

(f)

Fig. 5. Steady state performance of ASMC-IC-IPT and SMC-IC-IPT algorithm under grid voltage distortion: (a) Grid current. (b) Grid voltage and current for phase 'a'. (c) Grid voltage THD. (d) Load current THD. (e) Grid current THD using ASMC-IC-IPT. (f) Grid current THD using SMC-IC-IPT.

and (c) the 3-phase grid current, grid voltage and current for phase ' $a$ ' and the THD of distorted grid voltage is shown. The ASMC-IC-IPT scheme reduces the grid current THD from $29.35 \%$ to $2.80 \%$ and the SMC-IC-IPT scheme reduces the grid current THD to $3.38 \%$.

\section{Comparison Between the ASMC-IC-IPT and SMC-IC-IPT Schemes}

The THD of grid current and load current using the proposed ASMC-IC-IPT scheme are $2.62 \%$ and $27 \%$ and that of SMC-IC-IPT scheme is $3.24 \%$, which can be observed from Fig. 6(a), (b), and (c) respectively. The steady state response of grid current of ASMC-IC-IPT and SMC-IC-IPT scheme under ideal grid (without grid voltage distortion) are shown in Fig. 7(a). From Fig. 7(a) it is observed that, the grid current is made sinusoidal in $0.055 \mathrm{~s}$ using ASMC-ICIPT scheme, but it takes $0.08 \mathrm{~s}$ in SMC-IC-IPT scheme. The
ASMC-IC-IPT scheme shows fast response to that of SMCIC-IPT scheme under steady state condition. The transient response of grid current of ASMC-IC-IPT and SMC-ICIPT scheme is compared in Fig. 7(b). The response of grid current is shown during the time, the load current of phase ' $\mathrm{a}$ ' is disconnected from $0.5 \mathrm{~s}$ to $0.51 \mathrm{~s}$. The grid current is made sinusoidal instantly (just after connected to the load of phase 'a') using ASMC-IC-IPT scheme, but it takes $0.01 \mathrm{~s}$ to that of SMC-IC-IPT scheme. So it is observed that in ASMC-ICIPT scheme, the grid current adaptively made sinusoidal just after connection of the load of phase ' $a$ '.

\section{EXPERIMENTAL RESULTS}

The output power of solar simulator is fixed at $100 \mathrm{~W}$. The $\mathrm{dc}$ capacitor voltage is kept at $100 \mathrm{~V}$ fixed. The parameters used in the experimental setup are given in [8]. The picture of the experimental setup is shown in [8]. Performance 


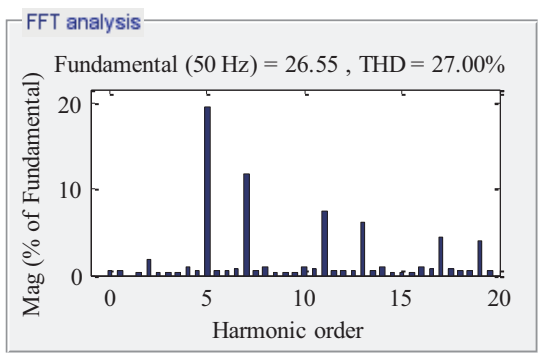

(a)

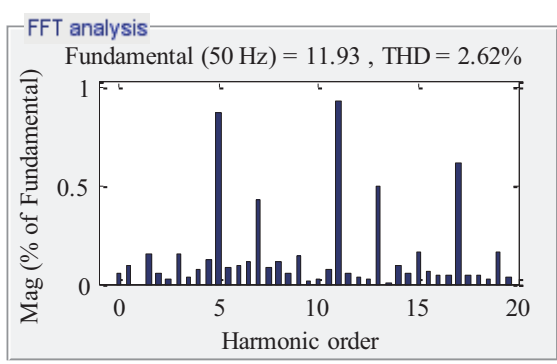

(b)

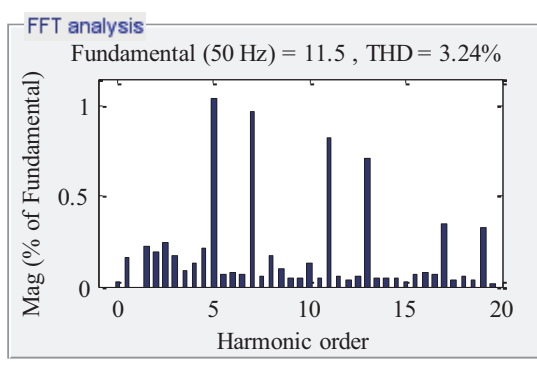

(c)

Fig. 6. Steady state performance comparison of ASMC-IC-IPT and SMC-IC-IPT: (a) Load current THD. (b) Grid current THD using ASMC-IC-IPT. (c) Grid current THD using SMC-IC-IPT.

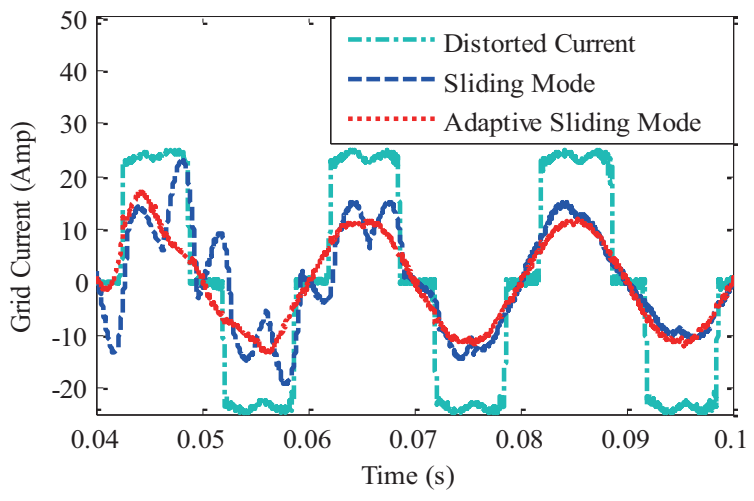

(a)

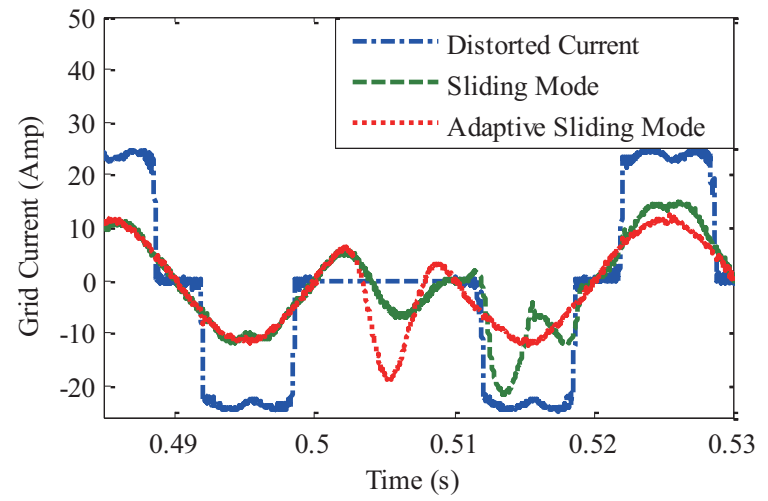

(b)

Fig. 7. Comparison of performance between ASMC-IC-IPT and SMC-IC-IPT: (a) Steady state grid current performance. (b) Grid current transient performance during disconnection of load current for phase ' $a$ '.

evaluation of the the modified IC algorithm was done for two cases, e.g. at $700 \mathrm{~W} / \mathrm{m}^{2}$ and $1000 \mathrm{~W} / \mathrm{m}^{2}$. In both the cases 98\% MPPT efficiency is achieved.

\section{A. Transient Performance of ASMC-IC-IPT}

The dynamic performance of ASMC-IC-IPT scheme using non-linear load I is shown in Fig. 8. Fig. 8(a) shows the grid voltage $v_{g a}$, grid current $i_{g a}$, load current $i_{L a}$, inverter current $i_{c a}$ of ASMC-IC-IPT scheme when non-linear load is added to phase ' $a$ '. It can be observed from Fig. 8(a) that, the grid current is sinusoidal even during the addition of non-linear load of phase 'a'. Fig. 8(b) shows the grid voltage $v_{g a}$, grid current $i_{g a}$, load current $i_{L a}$, inverter current $i_{c a}$ of ASMC-ICIPT scheme when there is a change in dc side resistance of non-linear load I. It can be observed from Fig. 8(b) that grid current, inverter current and load current change smoothly with the change in load resistance.

\section{B. Performance comparison of ASMC-IC-IPT and SMC-IC- IPT scheme}

The performance of ASMC-IC-IPT scheme is compared with that of the experimentation obtained from the SMCIC-IPT scheme. From Fig. 9(b), it can be observed that the dc capcitor voltage obtained from the SMC-IC-IPT scheme contains $5 \mathrm{~V}$ ripple. But $0.5 \mathrm{~V}$ ripples appeared in the case of ASMC-IC-IPT scheme as shown in Fig. 9(a). It can be observed from Fig. 10(a) and (b) that, there is 5\% overshoot in grid current $i_{g a}$ using ASMC-IC-IPT scheme and 25\% overshoot occurs in grid current $i_{g a}$ using SMC-IC-IPT scheme, when the APF is switched on.

\section{Performance of the Control Schemes}

SMC-IC-IPT: Time taken for grid current to make sinusoidal (during load disconnection): $0.01 \mathrm{~s}$, Time taken for grid current to make sinusoidal (under steady state): $0.09 \mathrm{~s}$, Grid current THD (in \%) under steady state with ideal grid (simulation): $3.24 \%$, Grid current THD (in \%) under steady state with grid voltage distortion (simulation): 3.38. Grid current THD (in \%) under steady state with ideal grid (experimental): $3.6 \%, \%$ of overshoot occured in grid current at the time of switching on the APF (experimental): $5 \%$, Presence of ripple in dc link voltage (experimental) in volt: $\pm 5 \mathrm{~V}$.

ASMC-IC-IPT: Time taken for grid current to make sinusoidal (during load disconnection): 0.003s, Time taken for grid current to make sinusoidal (under steady state): $0.06 \mathrm{~s}$, Grid current THD (in \%) under steady state with ideal grid (simulation): $2.62 \%$, Grid current THD (in \%) under steady state with grid voltage distortion (simulation): 2.80 , Grid current THD (in \%) under steady state with ideal grid (experimental): $2.9 \%, \%$ of overshoot occured in grid current at the time of 


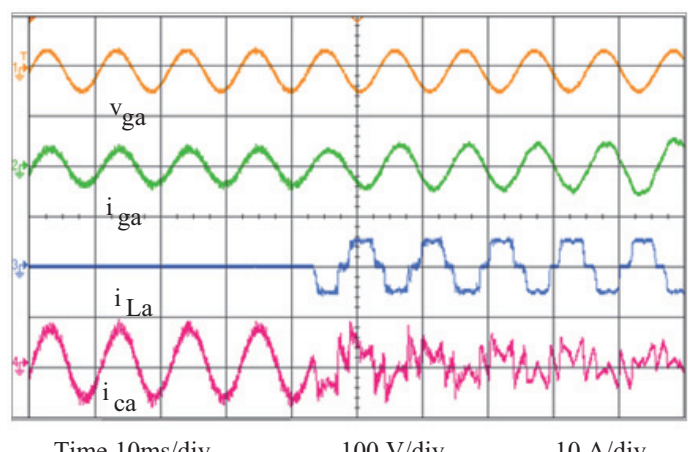

Time $10 \mathrm{~ms} / \mathrm{div}$

$100 \mathrm{~V} / \mathrm{div}$

$10 \mathrm{~A} / \mathrm{div}$

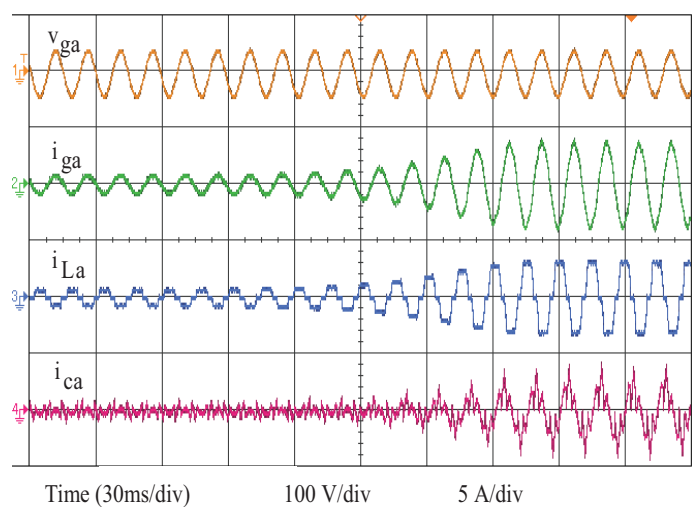

Fig. 8. Transient performance of ASMC-IC-IPT scheme during dynamic loading: (a) Performance during disconnection of load of phase 'a'. (b) Performance during change in dc side resistance of non-linear load I.

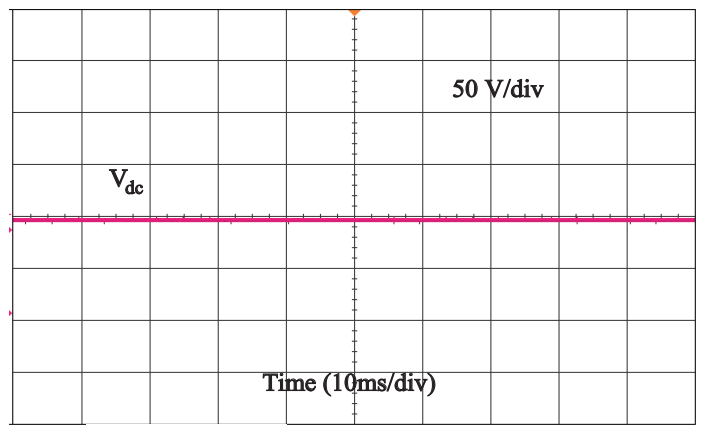

(a)

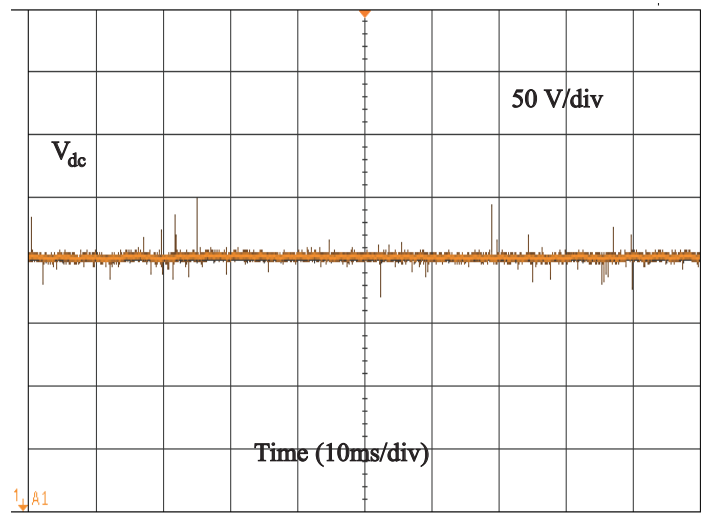

(b)

Fig. 9. Comparison of de link voltage between ASMC-IC-IPT and SMC-ICIPT scheme: (a) DC link voltage for ASMC-IC-IPT. (b) DC link voltage for SMC-IC-IPT.

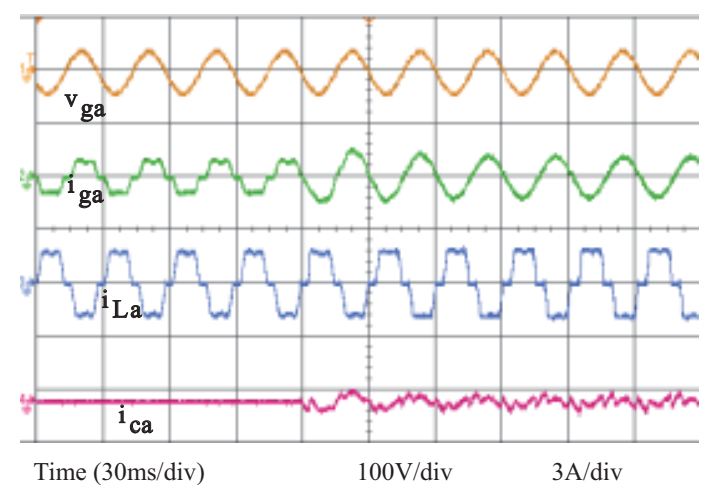

(a)

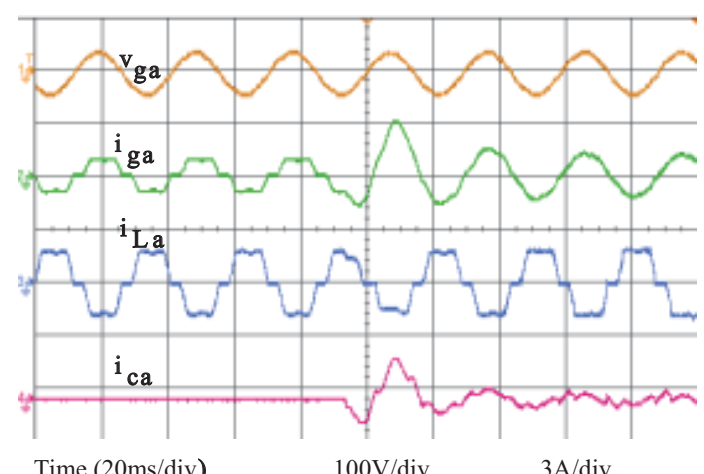

(b)

Fig. 10. Comparison of response between ASMC-IC-IPT and SMC-IC-IPT scheme when active power filter is switched on: (a) Grid voltage $v_{g a}$, grid current $i_{g a}$, load current $i_{L a}$, inverter current $i_{c a}$ of ASMC-IC-IPT. (b) Grid voltage $v_{g a}$, grid current $i_{g a}$, load current $i_{L a}$, inverter current $i_{c a}$, of SMC-IC-IPT.

switching on the APF (experimental): 5\%, Presence of ripple in de link voltage (experimental) in volt: $\pm 0.5 \mathrm{~V}$.

\section{CONCLUSIONS}

We proposed a new ASMC-IC-IPT controller for grid synchronization of a PV system, which involves a modified IC MPPT algorithm, to obtain max power from the PV panel during change in solar irradiance. Instantaneous power theory and power balance control theory are used to generate the reference inverter current. The simulation and experimental results of the proposed ASMC-IC-IPT scheme are compared with that of SMC-IC-IPT scheme. From the above results, it is observed that the proposed ASMC-IC-IPT control scheme exhibits robust and fast response even when there is distortion in the grid voltage, change in solar irradiance and dynamic loading conditions. This proposed controller also exhibits superior performance when compared with SMCIC-IPT controller in terms of reduced overshoot in grid current waveform $(25 \%$ to $5 \%)$ and ripples in dc capacitor voltage ( $5 \mathrm{~V}$ to $0.5 \mathrm{~V}$ ) using the experimental setup. Further, the proposed controller is efficient to bring down the THD of grid current from $3.24 \%$ to $2.62 \%$ in simulation (without grid voltage distortion), $3.38 \%$ to $2.80 \%$ (with grid voltage distortion) and $3.6 \%$ to $2.9 \%$ (without grid voltage distortion) in the experimental setup. The grid current THD 
is kept within the IEEE-519 standard.

\section{APPENDIX}

Considering the uncertainties due to parametric variations and measurements as described in section III-A, the SMC algorithm can be made adaptive by choosing a Lyapunov function as follows

$$
V(t)=\frac{1}{2} S^{2}+\frac{1}{2} \alpha\left(K_{1}-K_{1, \text { est }}\right)^{2}
$$

Taking time derivative of (34), we have

$$
\dot{V}(t)=S * \dot{S}-\alpha \bar{K}_{1} \dot{K}_{1, \text { est }}
$$

where, $K_{1}-\dot{K}_{1, \text { est }}=\widetilde{K}_{1}$ $\underset{\text { Taking }}{\text { obtain }} K_{1}=\frac{R_{c}}{L_{c}}$ and substituting (15) into (35) one can

$$
\dot{V}(t)=\mathrm{S}\left(\begin{array}{c}
\frac{d}{d t} I_{c d}^{*}-\frac{1}{L_{c}} D_{d} V_{p v}+K_{1} I_{c d} \\
-w I_{c q}+\frac{1}{L_{c}} V_{g d}+K_{d} e_{d} \\
-\alpha \bar{K}_{1} \dot{K}_{1, \text { est }}
\end{array}\right)
$$

Substituting (17) into (36), we have

$$
\begin{aligned}
\dot{V}(t) & =S\left[\frac{d}{d t} I_{c d}^{*}-\left(\frac{V_{p v}}{L_{c}}\right)\left\{\left(\frac{L_{c, \text { meas }}}{V_{p v, \text { meas }}}\right)\right)\left(\frac{d}{d t} I_{c d}^{*}\right.\right. \\
& +K_{d} e_{d, \text { meas }}+K_{d 1, \text { est }} \operatorname{sign}\left(S_{d}\right) \\
& \left.\left.+K_{1, \text { est }} I_{c d, \text { est }}-w I_{c q, \text { est }}+\frac{V_{g d, \text { meas }}}{L_{c d, \text { meas }}}\right)\right\} \\
+ & \left.K I_{c d}-w I_{c q}+\frac{V_{g d}}{L_{c}}+K_{d} e_{d}\right]-\alpha \widetilde{K}_{1} \dot{K}_{1, \text { est }}
\end{aligned}
$$

$K_{d 1}$ should be chosen that, the condition for SMC algorithm is satisfied, i.e.

$$
\frac{1}{2} \frac{d}{d t} S^{2} \leqslant-\eta|S|
$$

So taking $\theta=m_{\text {meas }} m^{-1}, K_{d 1}$ can be obtained as follows

$$
\begin{gathered}
K_{d 1} \geqslant(\theta-1)\left(\begin{array}{c}
K_{d, \text { est }} e_{d, \text { est }} \\
+I_{c d}^{*}+\frac{V_{g d, \text { meas }}}{L_{c, \text { meas }}} \\
-w I_{c q, \text { meas }} \\
+K_{1, \text { est }} I_{c d, \text { meas }}
\end{array}\right) \\
+\theta\left(\begin{array}{c}
\eta+K_{d} \Delta e_{d}+\Delta \frac{V_{g d}}{L_{c}} \\
-w \Delta I_{c q}+\Delta K_{1} I_{c d}
\end{array}\right) .
\end{gathered}
$$

The Lyapunov function will be negative definite if the tunning law for $\mathrm{d}$-axis will be as follows

$$
\dot{K}_{1, \text { est }}=\frac{I_{c d, \text { meas }} S}{\alpha} .
$$

Similarly taking $-\frac{R_{c}}{L_{c}}=K_{2}$ the value of $K_{q 1}$ can be obtained as follows

$$
\begin{gathered}
K_{q 2} \geqslant(\theta-1)\left(\begin{array}{c}
K_{q, e s t} e_{q, e s t} \\
+I_{c q}^{*} \\
+\frac{V_{g q, \text { meas }}}{L_{c, \text { meas }}} \\
-w I_{c d, \text { meas }} \\
+K_{2, \text { est }} I_{c q, \text { meas }}
\end{array}\right) \\
+\theta\left(\begin{array}{c}
\eta+K_{q} \Delta e_{q}+\Delta \frac{V_{g q}}{L_{c}} \\
-w \Delta I_{c d}+\Delta K_{2} I_{c q}
\end{array}\right) .
\end{gathered}
$$

The Lyapunov function will be negative definite using the tunning law for $q$-axis as follows

$$
\dot{K}_{2, e s t}=\frac{I_{c q, \text { meas }} S}{\alpha} .
$$

\section{REFERENCES}

[1] C. Meza, D. Biel, D. Jeltsema, and J. M. Scherpen, "Lyapunov-based control scheme for single-phase grid-connected pv central inverters," Teehrrol. IEEE Trans. Control. Syst., vol. 20, no. 2, pp. 520-529, Mar. 2012.

[2] I.-S. Kim, "Sliding mode controller for the single-phase gridconnected photovoltaic system," Applied Energy, vol. 83, no. 10, pp. 1101-1115, Oct. 2006.

[3] A. Abdelbaset, A.-H. M. El-Sayed, and A. E. H. Abozeid, "Grid synchro-nisation enhancement of a wind driven dfig using adaptive sliding mode control," IET Renewable Power Generation, vol. 11, no. 5, pp. 688-695, Mar. 2017.

[4] B. Subudhi and S. S. Ge, "Sliding-mode-observer-based adaptive slip ratio control for electric and hybrid vehicles," IEEE Trans. Intell. Transp. Syst, vol. 13, no. 4, pp. 1617-1626, 2012.

[5] Y. Zhu and J. Fei, "Adaptive global fast terminal sliding mode control of grid-connected photovoltaic system using fuzzy neural network approach," IEEE Access, vol. 5, pp. 9476-9484, May 2017.

[6] J. Fei and Y. Zhu, "Adaptive fuzzy sliding control of single-phase pv grid-connected inverter," PLOS one, vol. 12, no. 8, Aug. 2017.

[7] A. Mohan, D. Mathew, and V. M. Nair, "Grid connected pv inverter using adaptive total sliding mode controller," in Int Conf. on Control Communication and Computing, (13-15) Dec. 2013, Thiruvananthapuram, India. IEEE, 2013, pp. 457-462.

[8] A. Bag, B. Subudhi, and P. K. Ray, "Comparative analysis of sliding mode controller and hysteresis controller for active power filtering in a grid connected pv system," Int. J. Emerg. Elect. Power Syst., vol. 19, no. 1,2018

[9] N. D. Tuyen and G. Fujita, "Pv-active power filter combination supplies power to nonlinear load and compensates utility current," IEEE Power Energy. Technol Syst. J., vol. 2, no. 1, pp. 32-42, Mar. 2015. 


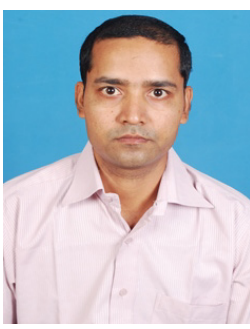

Aurobinda Bag received Bachelor degree in Electrical Engineering from Biju Patnaik University of Technology, Rourkela, Odisha, India, in 2005 and Master of Engineering degree in Power System Engineering from Veer Surendra Sai University of Technology (VSSUT), Burla, Odisha, India, in 2012. He has been awarded Ph.D. degree in Electrical Engineering from National Institute of Technology (NIT), Rourkela, Odisha, India in filtering and control of PV systems.

2019. His research interests include active power

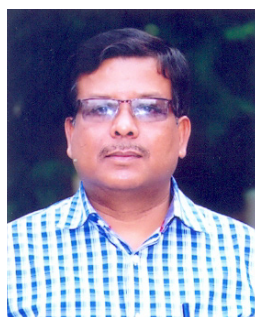

Bidyadhar Subudhi received Bachelor Degree in Electrical Engineering from National Institute of Technology (NIT), Rourkela, India, Master of Technology in Control and Instrumentation from Indian Institute of Technology, Delhi, India in 1988 and 1994 respectively and $\mathrm{PhD}$ degree in Control System Engineering from the university. of Sheffield, UK in 2003. He was a post doc in the Dept. of Electrical and Computer Engineering, National University of Singapore in 2005. Currently, he is a professor in the Department of Electrical Engineering at NIT Rourkela and coordinator, center of excellence on renewable energy systems. He is a Senior Member of IEEE and Fellow IET. His research interests include system and control theory, Control of PV Power System and Microgrid.

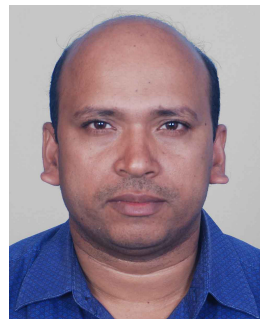

Pravat Kumar Ray received the B.E. degree in Electrical Engineering from Indira Gandhi Institute of Technology Sarang, Odisha, India, in 2000, the M.E. degree in Electrical Engineering from Indian Institute of Engineering Science and Technology, Shibpur, Howrah, India, in 2003, and the Ph.D. degree in Electrical Engineering from National Institute of Technology (NIT) Rourkela, Rourkela, India, in 2011. He was also a Postdoctoral Fellow at Nanyang Technological University, Singapore during Jan. 2016 to June 2017. He is currently an Associate Professor with the Department of Electrical Engineering, NIT Rourkela. His research interests include system identification, signal processing and soft computing applications to power system, power quality, solar irradiance forecasting using sky images and grid integration of renewable energy systems. 